\title{
Congruence lattices of pseudocomplemented semilattices
}

\author{
H. P. Sankappanavar
}

Congruence lattices of algebras in various varieties have been studied extensively in the literature. For example, congruence lattices (i.e. lattices of ideals) of Boolean algebras were characterized by Nachbin [18] (see also Gratzer [9] and Jonsson [16]) while congruence lattices of semilattices were investigated by Papert [19], Dean and Oehmke [4] and others.

In this paper we initiate the investigations into the structure of congruence lattices of pseudocomplemented semilattices. Our main concern here is two-fold: to develop certain indispensable tools, such as filter congruences, for the study of congruence lattices and to show that they possess several interesting (special) properties in addition to being algebraic.

After introducing the class of pseudocomplemented semilattices as a variety in Section 1 , a very special congruence $\Phi$ is defined. In Section 2 certain congruences, called filter congruences, are defined and then used to express every congruence as a join of two "simpler" congruences (in fact even better, see Theorem 2.6), a decomposition which is important for our subsequent investigations. In Section 3 we analyse the possibility of dually embedding a given pseudocomplemented semilattice in its congruence lattice, while Section 4 deals with several special properties of congruence lattices.

Most of the results in this paper form a part of the author's Ph.D. Thesis which was submitted to the University of Waterloo in 1974 and was written under the direction of Professor Stanley Burris. The author expresses his deep indebtedness and high appreciation to Professor Burris for his keen interest, constructive criticism and valuable remarks. The author is also grateful to Dr. S. BulmanFleming for several helpful conversations and for his interest in this paper. This work was essentially supported by an Ontario Graduate Fellowship and the final draft was prepared during January 1977 when the author was visiting the Department of Pure Mathematics, University of Waterloo and was supported by NRC Grant A7256.

Presented by G. Grätzer. Received March 22, 1977. Accepted for publication in final form March 21, 1978. 


\section{Preliminaries}

For the concepts and notations not defined in this paper as well as the results that are used here without proof the standard references are Birkhoff [2] and [3], Grätzer [8] and [9] and Jónsson [16].

An algebra $\langle S ; \wedge, *, 0\rangle$ is a pseudocomplemented semilattice iff $\langle S ; \wedge, 0\rangle$ is a $\wedge$-semilattice with least element 0 and $*$ is a unary operation on $S$ such that for $a \in S, a^{*}$ is the pseudocomplement of a in $S$, i.e. $x \wedge a=0$ in $S$ iff $x \leq a^{*}$. We shall denote by PCS the class of all pseudocomplemented semilattices; "PCS" will also be used as an abbreviation for "pseudocomplemented semilattice." For typographical convenience we shall identify an algebra with its universe when there is no confusion. Thus the PCS $\langle S ; \wedge, *, 0\rangle$ is abbreviated by "the PCS $S$."

It was shown by Balbes and Horn [1] that the class PCS is indeed a variety (equational class) whose defining identities, in addition to the three usual semilattice identities, are:

(1) $x \wedge 0=0$,

(2) $x \wedge(x \wedge y)^{*}=x \wedge y^{*}$

(3) $x \wedge 0^{*}=x$,

(4) $0^{* *}=0$.

$0^{*}$ being obviously the greatest element in a PCS will be denoted by 1 . Frink [6] has given a characterization of the class of Boolean algebras by a set of identities which are of the same similarity type as that of PCS and which include the defining identities of PCS, in other words the class of Boolean algebras, BA, is a subvariety of PCS. In fact, Jones [14] has shown that BA, viewed in this way, is the only nontrivial proper subvariety of PCS. In this work, unless otherwise stated, we regard Boolean algebras from this standpoint.

Frink [7] has shown that the following formulas - which will be useful for us - are true in PCS.

(5) $x \leq x^{* *}$,

(6) $x \leq y \rightarrow y^{*} \leq x^{*}$,

(7) $x \leq y \rightarrow x^{* *} \leq y^{* *}$,

(8) $x^{* * *}=x^{*}$,

(9) $x^{*} \wedge y^{*}=\left(x^{*} \wedge y^{*}\right)^{* *}$,

(10) $(x \wedge y)^{*}=\left(x^{* *} \wedge y^{* *}\right)^{*}$,

(11) $(x \wedge y)^{* *}=x^{* *} \wedge y^{* *}$.

It is also shown in Frink [7] that if $S$ is a PCS then the set of elements of the form 
$x^{*}, x \in S$, forms a Boolean algebra. We state this important structure theorem more precisely since we make frequent use of it, sometimes implicitly, in the sequel.

For a PCS $S$ we define $B(S)=\left\{x^{*}: x \in S\right\}$. The elements of $B(S)$ are called closed elements; equivalently $a \in S$ is closed iff $a=a^{* *}$. For $a \in S$, $a^{* *}$ is called the closure of $a$. We also define $N(S)=S-B(S)$, the elements of which will be referred to as non-closed.

THEOREM 1.1 (Frink [7]). If $S$ is a PCS then $\langle B(S), \wedge, \dot{V}, *, 0,1\rangle$ is a Boolean algebra (in the usual sense) such that $\left\langle B(S), \wedge,{ }^{*}, 0\right\rangle$ is a PCS-subalgebra of $S$ and the Boolean join $\dot{v}$ is defined by $a \dot{\vee} b=\left(a^{*} \wedge b^{*}\right)^{*}$.

The following corollary is immediate from the theorem.

COROLLARY 1.2. Let $S \in \mathrm{PCS}$. Then $S$ is a BA (in the sense of Frink) iff every element of $S$ is closed.

Unless otherwise stated, $S$ always represents an arbitrary fixed PCS.

We denote by Con $S$ the congruence lattice of $S$ whose least and greatest elements are denoted by $\Delta_{S}$ and $\nabla_{S}$ (sometimes simply $\Delta$ and $\nabla$ ) respectively. If $x \in S$ and $\psi \in$ Con $S$ then $[x] \psi$ or $[x]_{\psi}$ denotes the congruence class of $x$ modulo $\psi$; we simply write $[x]$ for $[x] \psi$ if no confusion is possible. If $H, K \subseteq S$ then we denote by $\theta(H \times K)$ the congruence generated by $H \times K \subseteq S \times S$; in particular, we shall denote by $\theta(a, b)$ the principal congruence generated by a pair $\langle a, b\rangle$ of elements of $S$.

It is shown in Frink [7] that the mapping **:S $\rightarrow S, s \rightarrow s^{* *}$ is a (PCS-) homomorphism. We denote by $\Phi(S)$ the kernel of this homomorphism, thus

$$
\Phi(S)=\left\{\langle x, y\rangle: x, y \in S \text { and } x^{* *}=y^{* *}\right\}
$$

or equivalently,

$$
\Phi(S)=\left\{\langle x, y\rangle: x, y \in S \text { and } x^{*}=y^{*}\right\}
$$

It is clear that $\Phi(S) \in$ Con $S$, and whenever there is no confusion we simply write $\Phi$ for $\Phi(S)$. It should be emphasized that we use the symbol $\Phi$ in this sense only throughout this paper. We also note that if $\theta \in \operatorname{Con} S$ and $a, b \in S$ then $\langle a, b\rangle \in$ $\Phi \vee \theta$ iff $\langle[a] \theta,[b] \theta\rangle \in \Phi(S / \theta)$.

The following remark is useful. 
Remark 1.3. Let $B$ be a Boolean algebra. Then the PCS-congruences on $B$ (as a PCS) are precisely the BA-congruences on $B$ (as a BA), because the join operation is a polynomial in $\wedge$ and *. Hence it is immaterial whether we regard Con $B$ as the lattice of BA-congruences on $B$ or as the lattice of PCScongruences on $B$. In particular, this applies to $B(S)$.

Recall that $B(S)$ is also a PCS-subalgebra of $S$. If $R$ is a relation on $S$, we denote by $(R)_{B}$ the restriction of $R$ to $B(S)$, i.e. $(R)_{B}=R \cap(B(S) \times B(S))$. The following lemma gives some useful properties of the operation of transition from $\theta$ to $(\theta)_{B}$.

LEMMA 1.4. Let $\alpha, \beta \in \operatorname{Con} S$. Then

(1) $(\Delta)_{B}=\Delta_{B}$ and $(\nabla)_{B}=\nabla_{B}$,

(2) $\alpha \subseteq \beta$ implies $(\alpha)_{B} \subseteq(\beta)_{B}$,

(3) $\alpha \subseteq \Phi$ iff $(\alpha)_{B}=\Delta_{B}$,

(4) $(\alpha)_{B} \subseteq \alpha$,

(5) $(\alpha \wedge \beta)_{B}=(\alpha)_{B} \wedge(\beta)_{B}$,

(6) $(\alpha \vee \beta)_{B}=(\alpha)_{B} \vee(\beta)_{B}=(\alpha)_{B} \circ(\beta)_{B}=(\alpha \circ \beta)_{B}$,

(7) $(\alpha \vee \Phi)_{B}=(\alpha)_{B}$.

Proof. (1)-(5) are immediate from the definitions. To prove (6) we first prove $(\alpha \circ \beta)_{B}=(\alpha)_{B} \circ(\beta)_{B}$. Suppose $x, y \in B(S)$ and $x \alpha s \beta y$ for some $s \in S$. Then $x \alpha s^{* *} \beta y$ since $x$ and $y$ are closed, and so $(\alpha \circ \beta)_{B} \subseteq(\alpha)_{B} \circ(\beta)_{B}$. Since the other inclusion is trivial, we thus have $(\alpha \circ \beta)_{B}=(\alpha)_{B} \circ(\beta)_{B}$. Now we know that

$$
\alpha \vee \beta=\bigcup_{\eta<\omega} \Lambda_{\eta}
$$

where

$$
\Lambda_{0}=\alpha, \quad \Lambda_{\eta+1}=\Lambda_{\eta} \circ \beta \circ \alpha
$$

and

$$
(\alpha)_{B} \vee(\beta)_{B}=\bigcup_{\eta<\omega} \Gamma_{\eta}
$$

where

$$
\Gamma_{0}=(\alpha)_{B}, \quad \Gamma_{\eta+1}=\Gamma_{\eta} \circ(\beta)_{B} \circ(\alpha)_{B} .
$$


Thus

$$
(\alpha \vee \beta)_{B}=\left(\bigcup_{\eta<\omega} \Lambda_{\eta}\right) \cap(B(S) \times B(S))=\bigcup_{\eta<\omega}\left(\Lambda_{\eta} \cap(B(S) \times B(S))\right) .
$$

An easy induction, using $(\alpha \circ \beta)_{B}=(\alpha)_{B} \circ(\beta)_{B}$, shows that $\Gamma_{\eta}=$ $\Lambda_{\eta} \cap(B(S) \times B(S))$ for all $\eta<\omega$, giving $(\alpha \vee \beta)_{B}=(\alpha)_{B} \vee(\beta)_{B}$. Finally, recalling that any two congruences on a Boolean algebra permute with each other, we get $(\alpha)_{B} \vee(\beta)_{B}=(\alpha)_{B} \circ(\beta)_{B}$ and thus (6) is proved. (7) follows immediately from (3) and (6).

\section{Filter congruences}

The notion of a filter in Boolean algebras can be easily generalized to PCS's. A non-empty subset $F$ of $S$ is a filter in $S$ iff $F$ is closed under $\wedge$, and if $a$ is in $F$ and $a \leq b$ then $b$ is in $F$. It is clear that the intersection of a family of filters in $S$ is also a filter in $S$. If $A \subseteq S$ then the filter $\langle A\rangle$ generated by $A$ is the intersection of filters in $S$ which contain $A$. If $s \in S$ then $[s, 1]$ is the principal filter generated by $s$.

With the help of the following lemma we will show that every filter in $S$ induces a congruence on $S$. We call such a congruence a filter congruence.

LEMMA 2.1. Let $s \in S$. Then $x \wedge s=y \wedge s$ implies $x^{*} \wedge s=y^{*} \wedge s$, for all $x, y \in S$.

Proof. Using (2) and the hypothesis we have

$$
\left(x^{*} \wedge s\right)=(x \wedge s)^{*} \wedge s=(y \wedge s)^{*} \wedge s=y^{*} \wedge s
$$

The above lemma is also due independently to J. T. Jones [14].

DEFINITION 2.2. Let $F$ be a filter in $S$. Define a relation $\hat{F}$, also denoted by $(F)^{\wedge}$, on $S$ as follows:

$\langle x, y\rangle \in \hat{F}$ iff there exists an $f \in F$ such that $x \wedge f=y \wedge f$.

We note here that the above definition is equivalent for Boolean algebras to:

$$
\langle x, y\rangle \in \hat{F} \quad \text { iff } \quad\left(x \vee y^{*}\right) \wedge\left(x^{*} \vee y\right) \in F
$$

LEMMA 2.3. If $F$ is a filter in $S$ then $\hat{F} \in$ Con $S$ and $\theta(F \times F)=\hat{F}=$ $\theta_{\text {semilar }}(F \times F)$. 
Remark 2.4. If $F=[a, 1]$ then $\hat{F}$ is denoted by $\hat{a}$ (or by $\left.(a)^{\wedge}\right)$ and

$$
\langle x, y\rangle \in \hat{a} \quad \text { iff } \quad x \wedge a=y \wedge a, \quad x, y \in S .
$$

LEMMA 2.5. If $F_{1}, F_{2}$ are filters in $S$ then $F_{1} \subseteq F_{2}$ implies $\hat{F}_{1} \subseteq \hat{F}_{2}$, and if $a, b \in S$ then $a \leq b$ implies $\hat{b} \subseteq \hat{a}$.

If $B$ is a $B A$ then the congruences on $B$ are, of course, precisely of the form $\hat{F}$ where $F$ is a filter in $B$. We shall now show that every congruence on a PCS $S$ can be expressed as the join of a congruence of the form $\hat{F}$, where $F$ is a filter on $S$, and a congruence contained in $\Phi$. In fact a much stronger statement is true.

THEOREM 2.6. (Decomposition Theorem ). Let $\psi \in$ Con $S$. Then

(i) $[1]_{\psi}$ is a filter in $S$,

(ii) $\psi=\left([1]_{\psi}\right)^{\wedge} \vee(\psi \wedge \Phi)=\left([1]_{\psi}\right)^{\wedge} \circ(\psi \wedge \Phi) \circ\left([1]_{\psi}\right)^{\wedge}$.

Proof. It is easy to show that $[1]_{\psi}$ is a filter in $S$.

To prove (ii), it is enough to prove that $\psi$ is contained in the extreme right hand expression. Let $\langle x, y\rangle \in \psi$. Then one can verify that $\left\langle\left(x^{*} \wedge y^{* *}\right)^{*}, 1\right\rangle \in \psi$, and by symmetry $\left\langle\left(x^{* *} \wedge y^{*}\right)^{*}, 1\right\rangle \in \psi$ and hence $\left(x^{*} \wedge y^{* *}\right)^{*} \wedge\left(x^{* *} \wedge y^{*}\right)^{*} \in[1]_{\psi}$. It is straightforward to verify $x^{* *} \wedge\left[\left(x^{*} \wedge y^{* *}\right)^{*} \wedge\left(x^{* *} \wedge y^{*}\right)^{*}\right]=$ $y^{* *} \wedge\left[\left(x^{*} \wedge\left[\left(x^{*} \wedge y^{* *}\right)^{*} \wedge\left(x^{* *} \wedge y^{*}\right)^{*}\right]\right.\right.$. Thus we get $\left\langle x^{* *}, y^{* *}\right\rangle \in\left([1]_{\psi}\right)^{\wedge}$ which implies that $\left\langle x, x \wedge y^{* *}\right\rangle \in\left([1]_{\psi}\right)^{\wedge}$ and $\left\langle x^{* *} \wedge y, y\right\rangle \in\left([1]_{\psi}\right)^{\wedge}$. Since $\langle x, y\rangle \in \psi$, we get $\left\langle x \wedge y^{* *}, x^{* *} \wedge y\right\rangle \in \psi$. Since by $(10)\left(x \wedge y^{* *}\right)^{*}=\left(x^{* *} \wedge y\right)^{*}$, we then obtain $\left\langle x \wedge y^{* *}, x^{* *} \wedge y\right\rangle \in \psi \wedge \Phi$ whereby one gets $\langle x, y\rangle \in\left([1]_{\psi}\right)^{\wedge} \circ\left([1]_{\psi}\right)^{\wedge}$, proving the theorem.

The following corollary - which is immediate from the preceding theorem shows that certain congruences on $S$, namely the congruences disjoint from $\Phi$, behave like the congruences on Boolean algebras in the sense that they are determined by a single congruence class, the class of 1 .

COROLLARY 2.7. Let $\psi \in$ Con $S$ be such that $\psi \wedge \Phi=\Delta$. Then $\psi=\left([1]_{\psi}\right)^{\wedge}$ where $[1]_{\Psi} \subseteq B(S)$.

We note here that the converse to this corollary is false.

In general if $\theta, \psi \in \operatorname{Con} S$ then $\theta \vee \psi$ cannot be expressed as a composition $\theta \circ \psi \circ \cdots \circ \theta$. Our next result, motivated by the proof of the preceding theorem, gives a simple, explicit expression for the join of two congruences in the case when one of the two is a filter congruence on $S$.

THEOREM 2.8. Let $F$ be a filter in $S$ and let $\psi \in$ Con $S$. Then $\hat{F} \vee \psi=$ $\hat{F} \circ \psi \circ \hat{F}$. 
Proof. It is sufficient to prove $\psi \circ \hat{F} \circ \psi \subseteq \hat{F} \circ \psi \circ \hat{F}$. Let $x \psi x_{1} \hat{F} x_{2} \psi y$. Then $x_{1} \wedge t=x_{2} \wedge t$ for some $t \in F$, and so $x \hat{F} x \wedge t \psi x_{1} \wedge t=x_{2} \wedge t \psi y \wedge t \hat{F} y$ and the proof is complete.

COROLLARY 2.9. Let $\beta \in \operatorname{Con} S$ with $\beta \wedge \Phi=\Delta$ and let $\psi \in$ Con $S$. Then $\beta \vee \psi=\beta \circ \psi \circ \beta$.

Easy examples show that Theorem 2.8 can not be improved further.

The following theorem gives a simple expression for the join of two congruences - when one of the two contains $\Phi$-in terms of their relative product.

THEOREM 2.10. Let $\beta, \psi \in$ Con $S$ with $\beta \supseteq \Phi$. Then $\beta \vee \psi=\beta \circ \psi \circ \beta$; in fact, $\beta \vee \psi=\beta \circ(\psi)_{B} \circ \beta$.

Proof. It suffices to show $\psi \circ \beta \circ \psi \subseteq \beta \circ(\psi)_{B} \circ \beta$. To this end, suppose $\langle x, y\rangle \in \psi \circ \beta \circ \psi$. Then $\left\langle x^{* *}, y^{* *}\right\rangle \in(\psi \circ \beta \circ \psi)_{B}$ and so, by Lemma 1.4 , we have $\left\langle x^{* *}, y^{* *}\right\rangle \in(\psi)_{B} \circ(\beta)_{B}$. But $\left\langle x, x^{* *}\right\rangle$ and $\left\langle y^{* *}, y\right\rangle$ are in $\beta$ since $\Phi \subseteq \beta$. Consequently

$$
\langle x, y\rangle \in \beta \circ(\psi)_{B} \circ(\beta)_{B} \circ \beta \subseteq \beta \circ(\psi)_{B} \circ \beta,
$$

as required.

\section{Dual embedding}

With the help of the congruences of the form $\hat{a}$ for a in $S$ we now establish that every PCS is "mirrored" inside its congruence lattice. Let $\hat{S}$ denote the set of congruences of the form $\hat{a}$ with $a$ in $S$.

THEOREM 3.1. There exists a dual PCS-isomorphism between $S$ and $\hat{S}$.

Proof. We shall show that the mapping $a \rightarrow \hat{a}$ is a dual PCS-isomorphism from $S$ onto $\hat{S}$.

First let us show that $(a \wedge b)^{\wedge}=\hat{a} \vee \hat{b}$. From Lemma 2.5 it is clear that $(a \wedge b)^{\wedge} \supseteq \hat{a} \vee \hat{b}$. On the other hand, let $\langle r, s\rangle \in(a \wedge b)^{\wedge}$. Then $r \wedge a \wedge b=s \wedge a \wedge b$ and so $r \wedge a \equiv s \wedge a(\hat{b})$. But $r \equiv r \wedge a(\hat{a})$ and $s \equiv s \wedge a(\hat{a})$, so $r \equiv s(\hat{a} \vee \hat{b})$, showing that $(a \wedge b)^{\wedge} \subseteq \hat{a} \vee \hat{b}$.

Now we show that if $a \in S$ then $\left(a^{*}\right)^{\wedge}$ is the dual pseudocomplement of $\hat{a}$ in Con $S$. First, observe that $\hat{a} \vee\left(a^{*}\right)^{\wedge}=\left(a \wedge a^{*}\right)^{\wedge}=\hat{0}=\nabla$. Next, let $\theta \in \operatorname{Con} S$ with $\hat{a} \vee \theta=\nabla$. Then by Theorem $2.8 \hat{a} \circ \theta \circ \hat{a}=\nabla$. Hence $\langle a, 0\rangle \in \hat{a} \circ \theta \circ \hat{a}$, so $a \equiv$ $x_{1}(\hat{a}), x_{1} \equiv x_{2}(\theta), x_{2} \equiv 0(\hat{a})$ for some $x_{1}, x_{2} \in S$. Thus $a=a \wedge x_{1} \theta a \wedge x_{2}=0$, i.e. 
$\langle a, 0\rangle \in \theta$ and hence $\left\langle a^{*}, 1\right\rangle \in \theta$, yielding $\left(a^{*}\right)^{\wedge} \subseteq \theta$, which shows that $\left(a^{*}\right)^{\wedge}$ is the dual pseudocomplement of $\hat{a}$. Thus we have shown that the mapping is a dual PCS-homomorphism.

The mapping is obviously onto. If $\hat{a}=\hat{b}$ then $\langle b, 1\rangle \in \hat{a}$ and so $a \wedge b=a$; by symmetry $a \wedge b=b$ wherefore $a=b$, showing that the mapping is one-one.

It is immediate from the above theorem that $|S| \leq \mid$ Con $S \mid$.

It should be pointed out that although $\hat{S}$ is dually pseudocomplemented as seen above, Con $S$ is not, in general, dually pseudocomplemented. The following theorem clarifies the situation.

THEOREM 3.2. Con $S$ is dually pseudocomplemented iff $B(S)$ is finite.

Proof. Let $B(S)$ be finite. We show that for every $\theta$ in Con $S$ the dual pseudocomplement of $\theta$ exists and is of the form â for some $a$ in $B(S)$. Since $(\theta)_{B}$ is a congruence on $B(S)$, the hypothesis implies that $(\theta)_{B}$ is a principal congruence and hence exists an element $c \in B(S)$ such that the filter $[c, 1]$ generates $(\theta)_{B}$. It is easy to verify that the dual pseudocomplement of $\theta$ is $\left(c^{*}\right)^{\wedge}$. Thus Con $S$ is dually pseudocomplemented. Now to show the converse, assume $B(S)$ is infinite. First observe that if $\theta^{+}$is a dual pseudocomplement of $\theta \in \operatorname{Con} S$ then $\left(\theta^{+}\right)_{B}$ is a dual pseudocomplement of $(\theta)_{B}$ in $\operatorname{Con} B(S)$, thus the hypothesis implies that Con $B(S)$ is also dually pseudocomplemented, which implies that the lattice of filters of the (infinite) $B A B(S)$ is dually pseudocomplemented, leading to a contradiction as it can be easily seen that a non-principal filter doesn't have a dual pseudocomplement.

COROLLARY 3.3. If $B(S)$ is finite then $B(S)$ is isomorphic to $B$ (dual of Con $S)$, the set of closed elements in the dual of Con $S$.

The following theorem - whose proof is easy and hence omitted-describes precisely when $\hat{S}$ of Theorem 3.1 coincides with Con $S$.

THEOREM 3.4. There is a dual PCS-isomorphism from $S$ onto Con $S$ iff either $S$ is a finite Boolean algebra, or else $S$ is of the form $B \cup\{1\}$ where $B$ is a finite BA and 1 is the new largest element of $S$.

It is well-known that the congruence lattice of a Boolean algebra determines, up to isomorphism, that Boolean algebra. However, for PCS's this is not the case, as the following example shows: 


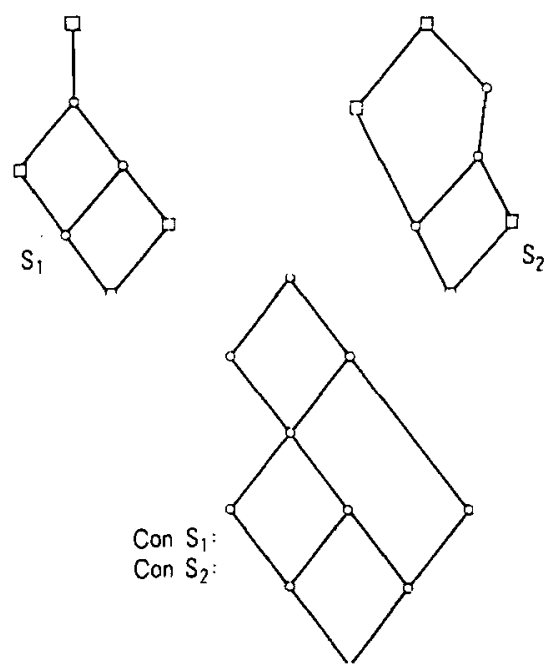

Fig. 1

\section{Example 3.5}

We conjecture that if $S$ satisfies the following property then Con $S$ determines $S$ upto isomorphism:

(U) $\forall x \forall y\left(x<y^{* *} \rightarrow\left(x^{*}=y^{*}\right.\right.$ or $\left.\left.x \leq y\right)\right)$.

\section{Properties of congruence lattices}

Congruence lattices of PCS's have, in addition to their being algebraic lattices, many interesting properties in common. In this section we shall give some such properties.

It is quite clear that Con $S$ is a complete sublattice of the lattice of semilatticecongruences on $S$; hence it follows immediately from Papert [19] that every interval in the congruence lattice of a PCS is pseudocomplemented. Then it is easy to see that $M_{5}$ cannot be embedded as a sublattice into the congruence lattice of a PCS (see Varlet [22]).

THEOREM 4.1. Con $S$ is coatomic, i.e. every $\theta \in \operatorname{Con} S$ is contained in some coatom of Con $S$. Furthermore, if $\theta$ is a coatom in Con $S$ then $\theta \supseteq \Phi$.

Proof. Observe that $\nabla=\theta(0,1)$ is compact in Con $S$ and use Zorn's lemma, while the second half is immediate from Lemma 1.4(7).

Remark 4.2. The congruence $\Phi$ gives rise to two important subintervals $[\Delta, \Phi]$ and $[\Phi, \nabla]$ in Con $S$ such that $\Phi$ is the intersection of all maximal congruences on $S$. 
A property of the congruence lattices of PCS's is contained in the following corollary to a well-known characterization of the congruence lattice of a Boolean algebra.

COROLLARY 4.3. The interval $[\Phi, \nabla]$ of Con $S$ is a distributive algebraic lattice in which the compact elements form a complemented sublattice. In particular, if $S$ is finite then $[\Phi, \nabla]$ is a (finite) BA.

(For a property of the interval $[\Delta, \Phi]$ see Corollary 4.10.)

Our next theorem gives another property of the congruences on a PCS and shows where the failure of distributivity can possibly occur.

THEOREM 4.4. Let $\beta, \gamma_{1}, \gamma_{2} \in$ Con $S$ such that either $\beta \subseteq \Phi$ or $\gamma_{1} \wedge \gamma_{2} \supseteq \Phi$. Then $\beta \vee\left(\gamma_{1} \wedge \gamma_{2}\right)=\left(\beta \vee \gamma_{1}\right) \wedge\left(\beta \vee \gamma_{2}\right)$.

Proof. Let $\beta \supseteq \Phi$. It is enough to show that the expression on the right is contained in the one on the left in the equation. Let $\langle x, y\rangle \in\left(\beta \vee \gamma_{1}\right) \wedge\left(\beta \vee \gamma_{2}\right)$, then $\left\langle x^{* *}, y^{* *}\right\rangle$ is in $\left((\beta)_{B} \vee\left(\gamma_{1}\right)_{B}\right) \wedge\left((\beta)_{B} \vee\left(\gamma_{2}\right)_{B}\right)=(\beta)_{B} \vee\left(\left(\gamma_{1}\right)_{B} \wedge\left(\gamma_{2}\right)_{B}\right)$ using Lemma 1.4 and the distributivity of $\operatorname{Con} B(S)$, hence $\left\langle x^{* *}, y^{* *}\right\rangle$ is in $(\beta \vee$ $\left.\left(\gamma_{1} \wedge \gamma_{2}\right)\right)_{B} \subseteq \beta \vee\left(\gamma_{1} \wedge \gamma_{2}\right)$. Since $\left\langle x, x^{* *}\right\rangle \in \beta$ and $\left\langle y^{* *}, y\right\rangle \in \beta$ by hypothesis, it follows that $\langle x, y\rangle \in \beta \vee\left(\gamma_{1} \wedge \gamma_{2}\right)$. The case $\gamma_{1} \wedge \gamma_{2} \supseteq \Phi$ is similar.

Remark 4.5. The above argument can be used to prove the following "complete version" of the above theorem:

Let $\beta, \gamma_{i} \in$ Con $S, i \in I$ such that either $\beta \supseteq \Phi$ or $\bigwedge_{i \in I} \gamma_{i} \supseteq \Phi$. Then $\beta \vee\left(\bigwedge_{i \in I} \gamma_{i}\right)=\bigwedge_{i \in I}\left(\beta \vee \gamma_{i}\right)$

If $A \subseteq S$, we define $(A)_{B}$ by $(A)_{B}=A \cap B(S)$.

LEMMA 4.6. Let $\gamma_{1}, \gamma_{2} \in$ Con $S$ be such that $\gamma_{i} \wedge \Phi=\Delta, i=1,2$ and $\left(\gamma_{1}\right)_{B}=$ $\left(\gamma_{2}\right)_{B}$. Then $\gamma_{1}=\gamma_{2}$.

Proof. Since $\gamma_{i} \wedge \Phi=\Delta$, we have by Corollary 2.7 that $\gamma_{i}=\left([1]_{\gamma_{i}}\right)^{\wedge}$, and $[1]_{\gamma_{i}}=$ $\left([1]_{\gamma_{i}}\right)_{B}, i=1,2$. From $\left(\gamma_{1}\right)_{B}=\left(\gamma_{2}\right)_{B}$ we get $\left([1]_{\gamma_{1}}\right)_{B}=\left([1]_{\gamma_{2}}\right)_{B}$ and hence $[1]_{\gamma_{1}}=$ $[1]_{\gamma_{2}}$ which implies $\gamma_{1}=\gamma_{2}$, proving the lemma.

Another property enjoyed by the congruence lattices of PCS's is given in the following theorem.

THEOREM 4.7. Let $\beta, \delta \in \operatorname{Con} S$ be such that $\beta \supseteq \Phi$. Then $[\delta \wedge \beta, \delta] \cong$ $[\beta, \delta \vee \beta]$. 
Proof. We shall first prove the theorem for the special case when $\delta \wedge \beta=\Delta$. With $\delta \wedge \beta=\Delta$ we will show $[\Delta, \delta] \cong[\beta, \delta \vee \beta]$. Define a mapping $f:[\Delta, \delta] \rightarrow[\beta, \delta \vee \beta]$ by $f(\gamma)=\gamma \vee \beta, \gamma \subseteq \delta$. We claim $f$ is an isomorphism. For, if $\gamma_{1}, \gamma_{2} \in[\Delta, \delta]$, then $f\left(\gamma_{1}\right)=\gamma_{1} \vee \beta$ and $f\left(\gamma_{2}\right)=\gamma_{2} \vee \beta$. Now obviously $f$ is a $v$ homomorphism. Also $f\left(\gamma_{1} \wedge \gamma_{2}\right)=\left(\gamma_{1} \wedge \gamma_{2}\right) \vee \beta=\left(\gamma_{1} \vee \beta\right) \wedge\left(\gamma_{2} \vee \beta\right)$ by Theorem 4.4 and hence $f\left(\gamma_{1} \wedge \gamma_{2}\right)=f\left(\gamma_{1}\right) \wedge f\left(\gamma_{2}\right)$ - thus $f$ is a lattice-homomorphism. Let $f\left(\gamma_{1}\right)=$ $f\left(\gamma_{2}\right)$. Then $\gamma_{1} \vee \beta=\gamma_{2} \vee \beta$ and so $\left(\gamma_{1}\right)_{B} \vee(\beta)_{B}=\left(\gamma_{2}\right)_{B} \vee(\beta)_{B}$. Also from $\delta \wedge \beta=\Delta$ and $\gamma_{i} \subseteq \delta, i=1,2$, we get $\gamma_{i} \wedge \beta=\Delta, i=1,2$ and so $\left(\gamma_{i}\right)_{B} \wedge(\beta)_{B}=\Delta_{B}$. Then using an argument for congruence lattices of Boolean algebras one can see that $\left(\gamma_{1}\right)_{B}=\left(\gamma_{2}\right)_{B}$. Also since $\gamma_{i} \wedge \beta=\Delta, i=1,2$ and $\beta \supseteq \Phi$, we get $\gamma_{i} \wedge \Phi=\Delta, i=1,2$. Thus applying Lemma 4.6 we get $\gamma_{1}=\gamma_{2}$ and hence $f$ is one-one. To show $f$ is onto, let $\psi \in[\beta, \delta \vee \beta]$. Then take $\gamma=\psi \wedge \delta$, and it is clear that $\delta \in[\Delta, \delta]$. Now $f(\gamma)=f(\psi \wedge \delta)=(\psi \wedge \delta) \vee \beta=(\psi \vee \beta) \wedge(\delta \vee \beta)$ by Theorem 4.4. Since $\beta \subseteq \psi \subseteq \delta \vee \beta$, we see that $f(\gamma)=\psi$, thus $f$ is onto. This shows that $f$ is an isomorphism.

Returning to the general case, we let $S_{1}=S / \delta \wedge \beta$ and let $\beta_{1}, \delta_{1}$ correspond $\beta, \delta$ respectively. Then $\beta_{1} \supseteq \Phi\left(S_{1}\right)$ and $\beta_{1} \wedge \delta_{1}=\Delta_{S_{1}}$, so from what we have just proved we get $\left[\Delta_{S_{1}}, \delta_{1}\right] \cong\left[\beta_{1}, \delta_{1} \vee \beta_{1}\right]$. Since $[\delta \wedge \beta, \delta] \cong\left[\Delta_{S_{1}}, \delta_{1}\right]$ and $\left[\beta_{1}, \delta_{1} \wedge \beta_{1}\right] \cong$ $[\beta, \delta \vee \beta]$, the theorem follows.

COROLLARY 4.8. If $\delta \in$ Con $S$ then $[\delta \wedge \Phi, \delta]$ is distributive.

Another property is given in the following.

THEOREM 4.9. Let $\beta, \delta \in \operatorname{Con} S$ such that $\delta \subseteq \Phi$. Then Con $S$ satisfies:

(B) If $\delta \succ \beta \wedge \delta$ then $\beta \vee \delta \succ-\beta$.

Proof. It suffices to show that $(B)$ holds in the congruence lattice of the quotient $S_{1}=S / \beta \wedge \delta$. Let $\beta_{1}, \delta_{1}$ denote the congruences on $S_{1}$ corresponding to $\beta, \delta$ respectively. Then $\beta_{1} \wedge \delta_{1}=\Delta_{S_{1}}$ and $\delta_{1}$ is an atom in Con $S_{1}$. Since $\delta \subseteq \Phi$ it is straightforward to verify that $\delta_{1}=\{\langle a, b\rangle,\langle b, a\rangle\} \cup \Delta$ for some $a, b \in S_{1}$ such that $a^{*}=b^{*}, b$ covers $a$ and every element of $S_{1}$ which is less than $b$ is already less than or equal to $a$. From this it follows immediately that $\delta_{1}$ is an atom in $\mathrm{Eq}\left(S_{1}\right)$, the lattice of equivalence relations on $S_{1}$. Since Con $S$ is a sublattice of Eq $\left(S_{1}\right)$ and the latter has the property $(B)$ the proof is complete.

\section{COROLLARY 4.10. $[\Delta, \Phi]$ has the property $(B)$.}

In view of the above corollary the height function is defined in the lattice $[\Delta, \Phi]$, and the following theorem relates the height of $\Phi$ with the number of non-closed elements in $S$. 
THEOREM 4.11. (i) $N(S)$ is finite iff $h(\Phi)$, the height of $\Phi$, is finite.

(ii) If $N(S)$ is finite then $h(\Phi)=n(S)$ where $n(S)$ is the cardinality of $N(S)$.

Proof. First part being obvious, for the second part we use induction on $n(S) \geq 1$. (Recall $N(S)=0$ iff $\Phi=\Delta$.) Let $n(S)=1$ and let $x$ be the unique non-closed element in $S$. Then it is easy to see that $\theta\left(x, x^{* *}\right)$ is an atom in Con $S$ and that $\Phi=\theta\left(x, x^{* *}\right)$, thus $h(\Phi)=1=n(S)$. Next, suppose that the theorem is true for all PCS's $S$ such that $n(S)=k$. Let $S$ be a PCS with $n(S)=k+1$. Observe that if $x \in N(S), y \in S, y<x^{* *}$ and $y \nless x$ then $y \wedge x \in N(S)$ and $(y \wedge x)^{* *}=y^{* *}$. Then it is easily verified, since $N(S)$ is finite, that there exists an $a \in N(S)$ such that whenever $y<a^{* *}$ one has $y<a$. Let $b \in S$ be such that $a \prec b$ and $a^{*}=b^{*}$ (the existence of such an element is clear. Then we claim that $z<b$ implies $z \leq a$ for every $z$ in $S$. For, if there is a $z \in S$ such that $z<b$ and $z \neq a$ then $z<a^{* *}$ and $z \nless a$, a contradiction. Thus the congruence $\theta(a, b)=\theta$ has one block consisting of exactly two elements $a, b$ and has all other blocks as singletons. Then $S_{1}=S / \theta$ has exactly $k$ non-closed elements and hence by the induction hypothesis $h\left(\Phi\left(S_{1}\right)\right)=$ $N\left(S_{1}\right)=k$. Since $\theta$ is an atom in $[\Delta, \Phi(S)]$ and $[\theta, \Phi(S)] \cong\left[\Delta_{1}, \Phi\left(S_{1}\right)\right]$ we conclude that $h(\Phi)=k+1$, completing the induction.

It is already stated in Section 1 that Boolean algebras are the only non-trivial subvariety of PCS. It is interesting to note that the class of congruence lattices of Boolean algebras, being distributive, satisfies every nontrivial lattice identity; the class of congruence lattices of PCS's, at the other extreme, does not satisfy any nontrivial lattice identity, a result which we proceed to derive from a similar result for congruence lattices of semilattices proved in [5] by Freese and Nation. We need the following lemma for the proof of this result.

Let $\mathscr{B}$ be a Boolean algebra (with universe $B$ ) and let Con $(\langle B, \wedge\rangle)$ denote the lattice of (semilattice) congruences on $\langle b, \wedge\rangle$ (as a semilattice, forgetting the other structure in $\mathscr{B})$. Let $S_{\mathrm{B}}$ denote $B \cup\{z\}$ where $z \notin B$ is the new least element. It is easily seen that $S_{B}$ is a PCS and $B\left(S_{B}\right)$ is a two-element BA. The following lemma is easily verified.

LEMMA 4.12. Let $\mathscr{B}$ be a BA. Then Con $(\langle B, \wedge\rangle)$ is a sublattice of Con $S_{B}$. In fact, Con $S_{B}$ is just Con $(\langle B, \wedge\rangle)$ with a new 1 adjoined.

We shall, for convenience, state the result due to Freese and Nation that was referred to earlier.

THEOREM 4.13. Let $\delta$ be a non-trivial lattice identity. Then there exists a finite Boolean algebra $\mathscr{B}(\delta)$ (with universe $B(\delta)$ ) such that $\delta$ fails in Con $(\langle B(\delta)$, $\wedge\rangle$. 
COROLLARY 4.14. Let $\delta$ be a non-trivial lattice identity. Then there exists $a$ finite PCS $\mathscr{S}(\delta)$ such that $\delta$ fails in Con $(\mathscr{S}(\delta))$. Thus the class of congruence lattices of PCS's does not satisfy any non-trivial lattice identity.

Proof. Take $S(\delta)$ to be $S_{\mathrm{B}(\delta)}$.

Finally the author would like to thank the referee for several useful comments.

\section{REFERENCES}

[1] R. Balbes and A. HoRn, Stone lattices, Duke Math. J. 37 (1970), 537-546.

[2] G. BIRKHOFF, Lattice Theory, 2nd ed. American Mathematical Society, 1948.

[3] G. BiRKHOF, Lattice Theory, 3rd ed. American Mathematical Society, 1967.

[4] R. A. DEAN and R. H. OEHMKE, Idempotent semigroups with distributive right congruence lattices, Pacific J. Math. 14 (1964), 1187-1209.

[5] R. Freese and J. B. NATION, Congruence lattices of semilattices, Pacific J. Math. 49 (1973), 51-58.

[6] O. Frink, Representations of Boolean algebras, Bull. Amer. Math. Soc. 47 (1941), 755-756.

[7] O. Frink, Pseudocomplements in semilattices, Duke Math. J. 29 (1962), 505-514.

[8] G. GrätZER, Universal Algebra, Van Nostrand, 1968.

[9] G. GRÄTZER, Lattice Theory, W. H. Freeman and Company, 1971.

[10] G. GRÄTZER and H. LAKSER, The structure of pseudocomplemented distributive lattices II. Congruence extension and amalgamation, Trans. Amer. Math. Soc. 156 (1971), 343-358.

[11] G. GRÄTZER and E. T. SCHMIDr, Ideals and Congruence relations in lattices, Acta Math. Acad. Sci. Hungar. 9 (1958), 137-175.

[12] T. E. HALL, On the lattice of congruences on a semilattice, J. Austral. Math. Soc. 12 (1971), 456-460.

[13] L. Henkin, J. D. Monk and A. TARsKi, Cylindric Algebras. Part I., North Holland, 1971.

[14] J. T. JoNES, Pseudocomplemented semilattices, Ph.D. Dissertation, U.C.L.A., 1972.

[15] J. T. JonEs, Projective pseudocomplemented semilattices, Pacific J. of Mathematics 52 (1974), 443-456.

[16] B. Jónsson, Topics in Universal Algebra, Springer-Verlag, 1972.

[17] T. KATRINÁK, Pseudocomplementare Halbverbände, Mat. Casopis Sloven. Akad. Vied. 18 (1968), 121-143.

[18] L. NACHBIN, On a characterization of the lattice of all ideals of a Boolean ring, Fund. Math. 36 (1949), 137-142.

[19] D. PAPERT, Congruence relations in semilattices, J. London Math. Soc. 39 (1964), 723-729.

[20] H. RAsiowa and R. SiKorski, The Mathematics of Metamathematics, Monog. Mat. Vol. 41, Warsaw, 1963.

[21] H. P. SankaPPanavar, A study of congruence lattices of pseudocomplemented semilattices, $\mathrm{Ph} . \mathrm{D}$. Thesis, University of Waterloo, 1974.

[22] J. VARLET, Congruence dans les demi-lattis, Bull. Soc. Roy. Sci. Liége, 34 (1965), 231-240.

Universidade Federal de Bahia Salvador, Bahia

Brazil

and

University of Waterloo

Waterloo, Ontario

Canada 